\title{
Cutaneous Precursor Lymphoid Neoplasm
}

National Cancer Institute

\section{Source}

National Cancer Institute. Cutaneous Precursor Lymphoid Neoplasm. NCI Thesaurus.

Code C45238.

A precursor lymphoid neoplasm that arises from the skin. 